\title{
Unlike calorie restriction, Roux-en-Y gastric bypass surgery does not increase hypothalamic AgRP and NPY in mice on a high-fat diet
}

\author{
Presheet P. Patkar ${ }^{1}$, Zheng Hao², Michael B. Mumphrey², R. Leigh Townsend ${ }^{2}$, Hans-Rudolf \\ Berthoud ${ }^{2}$, Andrew C. Shin 1 \\ ${ }^{1}$ Department of Nutritional Sciences, College of Human Sciences, Texas Tech University, \\ Lubbock, TX 79409, USA \\ ${ }^{2}$ Neurobiology of Nutrition Laboratory, Pennington Biomedical Research Center, Louisiana State \\ University System, Baton Rouge, LA 70808, USA
}

\begin{abstract}
Objectives: Dieting often fails because weight loss triggers strong counter-regulatory biological responses such as increased hunger and hypometabolism that are thought to be critically dependent on the master fuel sensor in the mediobasal hypothalamus (MBH). Because prolonged starvation has been shown to increase AgRP and NPY, the expression level of these two orexigenic genes has been taken as an experimental readout for the presence or absence of hunger. Roux-en-Y gastric bypass (RYGB) surgery leads to a significant weight loss without inducing the associated hunger, indicating possible changes in hypothalamic neuropeptides and/or signaling. Our goal was to assess key genes in the MBH involved in regulating body weight, appetite, and inflammation/ oxidative stress after RYGB surgery in mice.
\end{abstract}

Methods: Obese mice on a high-fat diet were subjected to either sham or RYGB surgery, or caloric restriction to match the weight of RYGB group. Chow-fed mice without surgery served as an additional control group. After 2 or 12 weeks post-surgery, hypothalamic genes were analyzed by real-time qPCR.

Results: During the rapid weight loss phase at 2 weeks after RYGB surgery, hypothalamic AgRP and NPY gene expression was not increased compared to mice with sham surgery, indicating that the mice are not hungry. In contrast, the same weight loss induced by caloric restriction promptly triggered increased AgRP and NPY expression. This differential effect of RYGB and caloric restriction was no longer observed during the weight maintenance phase at 12 weeks after surgery. A similar differential effect was observed for ObRb, but not for POMC and CART expression.

\footnotetext{
Users may view, print, copy, and download text and data-mine the content in such documents, for the purposes of academic research, subject always to the full Conditions of use:http://www.nature.com/authors/editorial_policies/license.html\#terms

Correspondence to: Andrew C. Shin, PhD, Department of Nutritional Sciences, College of Human Sciences, Texas Tech University, 2500 Broadway, Lubbock, TX 79409, Phone: (806) 834-1713, Fax: (806) 742-2926, andrew.shin@ttu.edu.

Competing Financial Interests

Authors declare no conflict of interest.

Supplementary information is available at the International Journal of Obesity website.
} 
Furthermore, RAGE and IBA-1, two markers for inflammation/oxidative stress, were significantly suppressed after RYGB compared to caloric restriction at 2 weeks post-surgery.

Conclusions: These findings suggest that RYGB prevents the biologically adaptive hunger response triggered by undernutrition and weight loss, and suppresses weight loss-induced hypothalamic inflammation markers.

\section{Introduction}

The ever-increasing obesity epidemic and related comorbidities such as Type II diabetes and heart disease are pushing obese individuals to undergo dieting through caloric restriction.

Unfortunately, many of them fail to continue dieting because the initial weight loss induces a strong drive of hunger and decreased energy expenditure as counter-regulatory responses for the body to restore its lost weight. The sense of hunger during starvation or caloric restriction is thought to be primarily mediated by a specific neuronal population in the mediobasal hypothalamus (MBH) through increased expression of orexigenic 'appetitestimulating' neuropeptides, agouti-related protein (AgRP) and neuropeptide Y (NPY) $)^{1,2}$. In this regard, the surprising absence of associated hunger drive in spite of a significant amount of weight loss following Roux-en-Y gastric bypass (RYGB) surgery ${ }^{3-5}$ strongly points to possible alterations in the expression of these critical neuropeptides. Others have previously investigated changes in hypothalamic gene expressions of several neuropeptides and hormone receptors following RYGB surgery, but the findings are not consistent. Vrang group $^{6}$ showed that RYGB surgery paradoxically increased mRNA of NPY and AgRP in the arcuate nucleus compared to sham-operated rats 8 weeks post-operatively, whereas others demonstrated no changes in POMC (proopiomelanocortin)/CART (cocaine/amphetamineregulated transcript) or NPY/AgRP 7,8 , or decreased hypothalamic NPY mRNA 3-8 weeks post-operatively ${ }^{9}$. More importantly, none of these studies included a weight-matched calorie-restricted group that is critical for dissecting the effect of weight loss independent of RYGB surgery.

We have recently developed a mouse model of RYGB surgery whose body weight and composition changes mirror those seen in humans with low mortality and complication rates ${ }^{10}$. The aim of the study was to utilize this mouse model, with addition of the important weight-matched group, to conduct a comprehensive mRNA analysis of key genes in the MBH involved in the regulation of appetite, body weight, inflammation, and oxidative stress at two time points - at 2 weeks post-surgery during the rapid weight loss phase, and at 12 weeks post-surgery when body weight has stabilized at a lower level.

\section{Materials and Methods}

\section{Animals}

Male diet-induced obese C57BL6J mice were purchased from Jackson Laboratories at the age of 18 weeks. Upon arrival at the Pennington Biomedical Research Center, they were continued on the same high-fat diet (60\% fat; $5.21 \mathrm{kcal} / \mathrm{g}$; Research Diets D12492) and also given access to regular chow (13\% fat; $3.36 \mathrm{kcal} / \mathrm{g}$; Purina 5001) for a total of 14 weeks. Animals were kept under standard laboratory conditions with a room temperature of 21- 
$23^{\circ} \mathrm{C}$ and a $12 \mathrm{~h}$ light-dark cycle (lights on at 07:00), with exceptions detailed below. Animal care and experimentation was approved by the Pennington Biomedical Research Center Institutional Animal Care and Use Committee and strictly followed rules and guidelines provided by the American Physiological Society and NIH.

\section{Experimental overview}

DIO mice were randomly stratified into 2 cohorts ( 2 and 12 week survival after surgery), each with 3 treatment groups with matching average body weight and fat mass [2 weeks: RYGB, $\mathrm{n}=13$; sham-surgery (sham, $\mathrm{n}=10$ ); no-surgery weight-matched controls (WM, $\mathrm{n}=$ 8); 12 weeks: RYGB, $n=9$; sham-surgery (sham, $n=10$ ); no-surgery weight-matched controls (WM, $\mathrm{n}=8)$ ]. All 6 groups were fed a two-choice diet consisting of high-fat and regular chow pellets for the duration of the study. While WM mice were given $65 \%$ of total caloric intake of RYGB mice in order for them to lose the equivalent amount of weight, 93\% of the calories came from HF diet and 7\% from regular chow diet, based on the food preference data we observed previously from RYGB mice ${ }^{10}$. In addition, age-matched control groups ( 2 weeks: Chow, $n=6 ; 12$ weeks, $n=4$ ) were fed only regular chow and were not subjected to any surgery. Sample size was estimated and chosen based on the previous phenotypic analysis ${ }^{10}$. Except for the time in the metabolic chambers, no other tests and invasive procedures were conducted that could perturb normal body weight regulation. At the end of the observation period, blood plasma and a large number of tissues were harvested for biochemical and histological analyses. Brains were extracted and immediately frozen with liquid nitrogen and stored at $-80^{\circ} \mathrm{C}$. Body weight, body composition, food intake, food choice, energy expenditure, and plasma levels of glucose, insulin, and leptin were reported previously ${ }^{10}$.

\section{RYGB surgery}

A detailed description of our mouse RYGB surgery can be found elsewhere ${ }^{11}$. Briefly, a 5-6 $\mathrm{cm}$ long Roux limb and a 6-7 cm long biliopancreatic limb were created by cutting the jejunum near the ligament of Treitz and anastomosing the distal end of the cut jejunum to a small gastric pouch and the proximal end with the mid-jejunum. Great care was taken to preserve gastric vessels and nerves near the cardia. Sham surgery consisted of midline laparotomy and gentle mobilization of stomach and intestines.

\section{Real-time (RT) quantitative PCR}

Animals were killed at the end of either 2 or 12 weeks after treatments. On the day of sacrifice, food was removed from all cages at 6am and mice were sacrificed between $10 \mathrm{am}-12 \mathrm{pm}$, resulting in 5 hours of fasting on average. Their brains were harvested, frozen immediately, and stored at $-80^{\circ} \mathrm{C}$. After sectioning the frozen brains in $200 \mu \mathrm{m}$ with a cryostat, the $\mathrm{MBH}$ comprising the arcuate nucleus and the ventromedial nucleus was dissected with micro-punch tools according to the mouse brain atlas ${ }^{12}$ and preserved in RNAlater (Thermo Fisher Scientific, Waltham, MA). The MBH punches were disrupted and homogenized in Qiagen lysis buffer using a sonicator (Qsonica, Newton, CT). Total RNA was extracted and purified using RNeasy Plus Universal Mini Kit (Qiagen, Germantown, MD) and the quality and quantity of RNA were measured using CYTATION 3 imaging reader (BioTek, Winooski, VT). cDNA was generated from $1 \mu \mathrm{g}$ total RNA using High 
Capacity cDNA Reverse Transcription Kit (Applied Biosystems, Foster City, CA) with the following reaction protocol in a thermal cycler (Mastercycler by Eppendorf, Hauppuage, $\mathrm{NY}$ ): $25^{\circ} \mathrm{C}$ for 10 minutes, $37^{\circ} \mathrm{C}$ for 120 minutes, $85^{\circ} \mathrm{C}$ for 5 minutes. The cDNA was diluted 1:20 with nuclease-free water and stored at $-20^{\circ} \mathrm{C}$. All primers were designed using the OligoArchitect primer design software and synthesized by Sigma Aldrich (St. Louis, $\mathrm{MO})$. Both no-RT and no-cDNA template negative controls showed absence of homo/ hetero-primer dimers and non-specific products at post-amplification. cDNA, at a final concentration of $5 \mathrm{ng} / \mu \mathrm{l}$, was amplified using iTaq Universal SYBR green Supermix (Biorad, Hercules, CA) on a CFX-Connect real-time PCR instrument (Biorad, Hercules, CA). Standard curves were prepared using serial dilutions of test cDNA at a primer-pair concentration of $450 \mathrm{nM}$ and the qPCR efficiency was confirmed for each primer pair. Amplification protocol for all genes was as follows: Initial denaturing at $95^{\circ} \mathrm{C}$ for 2 minutes followed by 40 cycles of denaturation at $95^{\circ} \mathrm{C}$ for 5 seconds, annealing at $60^{\circ} \mathrm{C}$ for 30 seconds, and completed with $95^{\circ} \mathrm{C}$ for 5 seconds, $65^{\circ} \mathrm{C}$ for 5 seconds and $95^{\circ} \mathrm{C}$ for 5 seconds. Melting curve analysis showed a single product for each primer pair. $\mathrm{Ct}$ values were determined using the regression fit module from CFX-Connect and final relative quantification was done using the delta-delta $\mathrm{Ct}$ method with Gapdh as the reference gene. Major neuropeptides (POMC, CART, BDNF, NPY, AgRP) and hormone receptors (ObRb, Y2R, GLP-1R, GHSR) important in appetite and body weight regulation, as well as markers for inflammation or oxidative stress (GFAP, NFkB, RAGE, TNF-a, IL-6, IL-1 $\beta$, IBA-1, ALCAM), were evaluated through RT-qPCR.

Primer sequences used are as follows: $P O M C$ - forward, AAGAGGTTAAGAGCAGTGA; reverse, ACATCTATGGAGGTCTGAAG; $C A R T$ - forward, GCAAGAAGTCCTGAAGAAG; reverse, GTAAAGACCGTGTGTAAGATT; $N P Y-$ forward, CTAGGTAACAAGCGAATGG; reverse, GGTGATGAGATTGATGTAGTG; $A g R P$ - forward, ACAAGAGACCAGGACATC; reverse, CAACAGCAGAACACAACT; $G L P-1 R$ (glucagon-like peptide 1 receptor) - forward, TGGCTACATAAGGACAACT; reverse, CAACAAGGATGGCTGAAG; $Y 2 R$ (peptide $Y Y$ receptor) - forward, GCAGAGGCAGATGAGAAT; reverse, AAGATGATGGAGCAGTAGG; $B D N F$ (brainderived neurotrophic factor) - forward, TTGGAAGCCTGAATGAATG; reverse, CCTAAGATGAAGCGATTGTT; $O b R b$ - forward, AGTCACAAGATAATGGAGAATAAG; reverse, GGCTCTACTGGAATGGAA; GHSR - forward, GAGCGTCTTCTTCTTCCT; reverse, AGCATCTTCACTGTCTGTT; TNF- $a$ - forward, CCACCATCAAGGACTCAA; reverse, ATCTTATCCAGCCTCATTCT; $I L-6$ - forward, ACAGAAGGAGTGGCTAAG; reverse, AGAGAACAACATAAGTCAGATAC; $I L-1 \beta$ - forward, GCAGCACATCAACAAGAG; reverse, CAGCAGGTTATCATCATCATC; $N F-k B-$ forward, AAGAAGGACACGACAGAA; reverse, CAGGCTATTGCTCATCAC; GFAPforward, CGCATCACCATTCCTGTA; reverse, GGAGCACGCATCTATCTAA; $R A G E-$ forward, CTACCGAGTCCGAGTCTA; reverse, CCTGGTCTCTTCCTTCAC; ALCAMforward, GAGGCAGACGATATAAGTGA; reverse, GAGGAGACCAACGACAAT; IBA-1 forward, TCTGCCATCTTGAGAATGA; reverse, TGACATCCACCTCCAATC. 


\section{Statistical analysis}

Experimenters were blind to the groups during data analysis. mRNA abundance between 4 groups was first normalized to GAPDH and expressed as fold change from the chow-fed lean control group. This was analyzed by ANOVA followed by Bonferroni's post hoc multiple comparison tests. Body weight and caloric intake data were analyzed by repeated ANOVA followed by Bonferroni's post hoc multiple comparison tests. Data are presented as Mean \pm SEM. Statistically significant difference was set at $p<0.05$.

\section{Results}

\section{Effects on body weight, body composition, and food intake}

The effects on comprehensive metabolic parameters from the 12-week cohort were reported previously ${ }^{10}$. In this cohort, RYGB reduced body weight by about $17 \%$ compared to presurgical levels and $34 \%$ compared to sham-operated mice, mostly owing to fat mass loss. There was a small mean weight regain of $5 \%$ from the nadir that occurred at about 3 weeks. In the 2-week cohort, RYGB reduced body weight by about $22 \%$ compared to pre-surgical levels and 18\% compared to sham-operated mice (Supplemental Fig. S1). Plasma leptin levels at both 2 and 12 weeks of both RYGB and WM mice were much lower than sham mice and not significantly different from chow-fed control mice and from each other ${ }^{10}$. Food intake was significantly lower for the first 8 days after RYGB, and then recovered to near pre-surgical levels after 2 weeks (Supplemental Fig. S2).

\section{Effects on gene expression toward the end of the weight loss phase}

At 2 weeks, mRNA expression of AgRP and NPY were significantly higher in calorierestricted but not in mice with RYGB, compared to sham-operated mice (Fig. 1A). There was a similar trend with ghrelin receptor (GHSR) expression that did not reach statistical significance. Compared to chow-fed control mice without any surgery, expression levels of AgRP, NPY, and GHSR were significantly lower in both RYGB and sham-operated mice but not in calorie-restricted WM mice.

Among the anorexigenic genes assessed, only leptin receptor $(\mathrm{ObRb})$ expression was differentially expressed between calorie-restricted and RYGB mice (Fig. 1A). However, somewhat unexpectedly, ObRb expression in calorie-restricted WM mice was significantly higher compared to RYGB mice. There were no significant differences in mRNA expression of the anorexigenic factors POMC and CART among the 4 groups. However, BDNF mRNA was drastically reduced in all 3 groups fed a high-fat diet. Y2R mRNA was significantly lower in the RYGB compared to lean chow-fed controls, while there were no significant differences in GLP-1R expression.

Expression of genes related to inflammation or oxidative stress including TNF-a, RAGE, IL-6, IBA-1, and NFkB (Fig. 2) was generally lowest in mice with RYGB, but not significantly lower than in sham-operated mice. With the exception of TNF-a, none of these genes were upregulated in sham-operated obese mice. The 2.2-fold upregulation of TNF-a was however not statistically significant due to a high variability. Interestingly, RAGE and 
IBA-1 mRNA was significantly higher in WM group that lost the same amount of weight as RYGB mice.

\section{Effects on gene expression during the weight maintenance phase}

Most of the differences in the expression of orexigenic genes observed at 2 weeks were no longer observed at 12 weeks, with the exception of NPY, which was significantly lower in sham/obese mice compared to chow-fed controls (Fig. 1B). However, expression of AgRP, NPY, and GHSR was similar between calorie-restricted and RYGB mice.

Among the anorexigenic genes assessed, POMC was significantly increased in sham vs. RYGB and chow controls (Fig. 1B). Also, there was a trend for increased BDNF in all three high-fat fed groups compared to chow controls, opposite to what we observed at 2 weeks.

Similarly, except for IBA-1 and ALCAM, TNF- $a$, IL-6, IL-1 $\beta$ and NFkB mRNA was no longer upregulated in WM controls (Fig. 2).

\section{Discussion}

Orexigenic NPY/AgRP and anorexigenic POMC/CART-expressing neurons located in the MBH play a critical role in controlling body weight and food intake as they are able to sense nutrients and related signals such as leptin, insulin, and GLP- ${ }^{13}$. Eating to satiety ${ }^{1,14}$ has been shown to stimulate POMC and CART gene expression and inhibit NPY and AgRP expression. Conversely, in a state of nutrient deficit such as mild caloric restriction $(30 \%)^{15}$, food deprivation (24-48h) ${ }^{1,14}$ or longer starvation (4-6 days) with significant weight loss $^{2}, 16$, NPY and AgRP expression is induced while that of POMC and CART is suppressed. Given that RYGB surgery lowers drive of hunger even after a profound weight loss ${ }^{3-5}$, we would predict lower NPY and AgRP mRNA, and perhaps higher expression of POMC and CART. In contrast, their expression is expected to increase during caloric restriction-induced weight loss, indicating greater hunger and craving. Indeed, our results extend the previous findings ${ }^{6-9}$ and demonstrate that RYGB induces changes in hypothalamic genes that are distinct from those in caloric-restricted, weight-matched controls, suggesting weight loss-independent effects. More interestingly, many of these RYGB-specific differences disappear during the chronic weight-maintenance phase in spite of the sustained body weight loss.

As expected, mice weight-matched to RYGB by caloric restriction had increased gene expressions of NPY and AgRP compared to sham obese group, suggesting that they were "hungry" and eager to restore their original body weight. Unlike the calorie-restricted WM mice, RYGB mice did not upregulate NPY and AgRP expression, suggesting that they were not "hungry" and willing to restore their pre-surgical body weight. This is supported by their food intake that is near pre-surgical levels but not higher to gain back their lost weight. Kawasaki group has shown similar findings after sleeve gastrectomy in HF-fed rats ${ }^{17}$. Given that mice with RYGB lose about $1-3 \mathrm{kcal} /$ day of energy in the feces ${ }^{18,19}$, their food intake may not quite be restored at the end of 2 weeks. Thus, weight loss induced by RYGB provoked opposite responses in AgRP and NPY mRNA, consistent with the notion that RYGB surgery establishes a lower body weight set-point. In line with others' findings ${ }^{20}$, 
sham-operated, obese mice displayed lower NPY and AgRP mRNA compared to chow-fed lean controls, reflecting a state of positive energy balance. On the other hand, anorexigenic POMC and CART mRNA was not different between groups which is in agreement with findings by Grayson and colleagues ${ }^{7}$. While the reason is not clear, an earlier study by the Meguid lab ${ }^{21}$ has shown increased immunostaining of a-MSH, an anorexigenic peptide posttranslationally cleaved from POMC, following RYGB surgery. It is thus possible that in our study, RYGB in obese mice raises the melanocortinergic tone, which is not reflected in POMC mRNA, to counter the hunger drive associated with weight loss. This may also explain lower AgRP and NPY mRNA compared to those in WM group. This warrants further investigation.

Reduced Y2R in RYGB mice compared to chow-fed lean controls may be interpreted as a compensatory downregulation due to over-activation of the receptor by the anorexigenic gut hormone PYY. Although not measured in this study, our ${ }^{22}$ and others' previous work ${ }^{23}$ clearly demonstrated substantially higher basal and/or meal-stimulated plasma PYY levels after RYGB surgery. This may also help explain lower NPY/AgRP mRNA expression in spite of dramatic weight loss in these mice. The same potential mechanism does not seem to apply for lower ObRb in RYGB mice since their plasma leptin levels were low and comparable to that of both lean and WM control groups.

About half of the genes related to inflammation or oxidative stress were reduced in sham obese mice compared to chow-fed lean controls, although not reaching statistical significance for the most part. While HF feeding or diet-induced obesity is often associated with hypothalamic inflammation ${ }^{24}$, our data are in agreement with others' findings that showed either no change or even decreased hypothalamic inflammatory markers in dietinduced obese mice 25,26 . The discrepancy may be explained by the duration of HF feeding and/or HF diet composition. Importantly, mRNA of these markers were similarly lowered in RYGB but not WM group, suggesting that WM mice might perceive restricted feeding and the subsequent weight reduction as stressors while RYGB mice do not. It would be critical to consider these data with proteomics and metabolomics analyses, among others, in near future to better understand if RYGB surgery indeed alters hypothalamic inflammatory state.

It is important to mention that while food was removed from all cages $5 \mathrm{~h}$ before sacrifice, we observed that calorie-restricted WM mice finished their reduced food ration beforehand sometime during the second half of the dark phase, resulting in a fasting duration of about $6-10 \mathrm{~h}$ rather than $5 \mathrm{~h}$ as intended. Therefore, this longer fasting duration in WM mice could have potentially contributed to the differences in our gene expression data. However, two important considerations suggest that this difference in fasting duration likely was not responsible for the large observed differences in gene expression. First, AgRP and NPY gene regulation is known to be driven primarily by the overall energy status of an organism (ie. body weight, fat mass) and not by a relatively acute, short-term fasting. In support of this notion, many studies have shown that at least $48 \mathrm{~h}$ fasting or longer that leads to marked reduction in body weight significantly increases AgRP and/or NPY mRNA $2,14,16,27-32$. Boswell and colleagues have shown that $18 \mathrm{~h}$ fasting was sufficient to induce changes in gene expression ${ }^{33}$, which is still longer than the potential 10h fasting with WM mice. Only one group has demonstrated that NPY mRNA was significantly increased by over 2 -fold 
after only $4 \mathrm{~h}$ fasting, but that level came down by $8 \mathrm{~h}$ fasting ${ }^{34}$. More convincing evidence comes from a study by $\mathrm{Xu}$ and colleagues ${ }^{35}$ that showed that rats undergoing food restriction without weight loss do not show a significant increase of NPY mRNA up to 16h of fasting, indicating that NPY/AgRP genes do not change much due to a moderate fasting if it does not involve a significant weight loss. Collectively, these studies suggest that NPY/AgRP mRNA is more sensitive to long-term energy status, that is, to a reduction in weight or fat mass, but not to short-term fasting. Second, leptin is an adipocyte-derived hormone whose plasma level is sensitive to short-term fluctuations in nutritional state. This is supported by studies demonstrating a significant drop of plasma leptin only after 4-6h fasting 34, 36, 37 . The fact that plasma leptin levels in RYGB and WM mice in our study were essentially identical at the time of sacrifice ( $\mathrm{ng} / \mathrm{ml} \pm$ SEM; $4.5 \pm 0.7 \mathrm{RYGB}$ vs. $4.8 \pm 1.3 \mathrm{WM}$ ) ${ }^{10}$ strongly indicates similar nutritional status between these groups, in other words, a similar fasting state. Thus, although we cannot rule out different fasting durations in WM vs. other groups, we think it is very unlikely that few more hours of fasting in WM mice would have caused the differences in gene expression we observed in this study. Rather, our findings are consistent with the idea that RYGB surgery suppresses the adaptive hunger response.

Sham and RYGB mice show divergent body weight curves during the acute phase while sharing similar mRNA levels for many genes examined here, suggesting that these genes may serve different roles after surgery. Interestingly, most of the changes were no longer present during the chronic phase when RYGB mice were successfully sustaining their weight loss. This may indicate reduced sensitivity to RYGB-induced signaling mechanisms. Alternatively, there may be a group of genes important for "lowering" vs. "maintaining" body weight and fat mass within and/or outside the MBH. Given the reduction of BDNF mRNA among treatment groups in the acute phase and its concerted return to the presurgical levels in the chronic phase, we speculate that BDNF may be one of the genes that help maintain new body weight and fat mass.

In conclusion, we provide evidence demonstrating that RYGB surgery differentially regulates hypothalamic genes critical for appetite, body weight, and inflammation/oxidative stress during the acute "rapid weight-loss" phase and chronic "weight maintenance" phase. Importantly, our findings show that weight loss induced by caloric restriction but not RYGB increases the expression of orexigenic and inflammatory genes, suggesting that RYGB surgery suppresses the adaptive hunger and stress response to weight loss. Our results further show that these changes disappear during the weight-maintenance / weight-regain phase, possibly indicating development of tolerance to the RYGB-induced signaling mechanisms.

\section{Supplementary Material}

Refer to Web version on PubMed Central for supplementary material.

\section{Acknowledgements}

This study was supported by NIH DK099463 (ACS) and NIH DK047348 (HRB). 


\section{References}

1. Swart I, Jahng JW, Overton JM, Houpt TA. Hypothalamic NPY, AGRP, and POMC mRNA responses to leptin and refeeding in mice. Am J Physiol Regul Integr Comp Physiol 2002; 283(5): R1020-6. [PubMed: 12376393]

2. Bertile F, Oudart H, Criscuolo F, Maho YL, Raclot T. Hypothalamic gene expression in long-term fasted rats: relationship with body fat. Biochem Biophys Res Commun 2003; 303(4): 1106-13. [PubMed: 12684050]

3. Karamanakos SN, Vagenas K, Kalfarentzos F, Alexandrides TK. Weight loss, appetite suppression, and changes in fasting and postprandial ghrelin and peptide-YY levels after Roux-en-Y gastric bypass and sleeve gastrectomy: a prospective, double blind study. Ann Surg 2008; 247(3): 401-7. [PubMed: 18376181]

4. Morinigo R, Moize V, Musri M, Lacy AM, Navarro S, Marin JL et al. Glucagon-like peptide-1, peptide YY, hunger, and satiety after gastric bypass surgery in morbidly obese subjects. J Clin Endocrinol Metab 2006; 91(5): 1735-40. [PubMed: 16478824]

5. Scholtz S, Miras AD, Chhina N, Prechtl CG, Sleeth ML, Daud NM et al. Obese patients after gastric bypass surgery have lower brain-hedonic responses to food than after gastric banding. Gut 2014; 63(6): 891-902. [PubMed: 23964100]

6. Barkholt P, Pedersen PJ, Hay-Schmidt A, Jelsing J, Hansen HH, Vrang N. Alterations in hypothalamic gene expression following Roux-en-Y gastric bypass. Mol Metab 2016; 5(4): 296304. [PubMed: 27069869]

7. Grayson BE, Hakala-Finch AP, Kekulawala M, Laub H, Egan AE, Ressler IB et al. Weight loss by calorie restriction versus bariatric surgery differentially regulates the hypothalamo-pituitaryadrenocortical axis in male rats. Stress 2014; 17(6): 484-93. [PubMed: 25238021]

8. Cavin JB, Voitellier E, Cluzeaud F, Kapel N, Marmuse JP, Chevallier JM et al. Malabsorption and intestinal adaptation after one anastomosis gastric bypass compared with Roux-en-Y gastric bypass in rats. Am J Physiol Gastrointest Liver Physiol 2016; 311(3): G492-500. [PubMed: 27418681]

9. Liu JY, Mu S, Zhang SP, Guo W, Li QF, Xiao XQ et al. Roux-en-Y gastric bypass surgery suppresses hypothalamic PTP1B protein level and alleviates leptin resistance in obese rats. Exp Ther Med 2017; 14(3): 2536-2542. [PubMed: 28947917]

10. Hao Z, Mumphrey MB, Townsend RL, Morrison CD, Munzberg H, Ye J et al. Body Composition, Food Intake, and Energy Expenditure in a Murine Model of Roux-en-Y Gastric Bypass Surgery. Obes Surg 2016; 26(9): 2173-2182. [PubMed: 26781597]

11. Hao Z, Zhao Z, Berthoud HR, Ye J. Development and verification of a mouse model for Roux-enY gastric bypass surgery with a small gastric pouch. PLoS One 2013; 8(1): e52922. [PubMed: 23326365]

12. Paxinos G, Franklin K. The mouse brain in streotaxic coordinates, 2nd edn Academic Press, 2004.

13. Clemmensen C, Muller TD, Woods SC, Berthoud HR, Seeley RJ, Tschop MH. Gut-Brain CrossTalk in Metabolic Control. Cell 2017; 168(5): 758-774. [PubMed: 28235194]

14. Beck B, Jhanwar-Uniyal M, Burlet A, Chapleur-Chateau M, Leibowitz SF, Burlet C. Rapid and localized alterations of neuropeptide $\mathrm{Y}$ in discrete hypothalamic nuclei with feeding status. Brain Res 1990; 528(2): 245-9. [PubMed: 2271925]

15. Shimokawa I, Fukuyama T, Yanagihara-Outa K, Tomita M, Komatsu T, Higami Y et al. Effects of caloric restriction on gene expression in the arcuate nucleus. Neurobiol Aging 2003; 24(1): $117-$ 23. [PubMed: 12493557]

16. Brady LS, Smith MA, Gold PW, Herkenham M. Altered expression of hypothalamic neuropeptide mRNAs in food-restricted and food-deprived rats. Neuroendocrinology 1990; 52(5): 441-7. [PubMed: 2177853]

17. Kawasaki T, Ohta M, Kawano Y, Masuda T, Gotoh K, Inomata M et al. Effects of sleeve gastrectomy and gastric banding on the hypothalamic feeding center in an obese rat model. Surg Today 2015; 45(12): 1560-6. [PubMed: 25724939]

18. Nestoridi E, Kvas S, Kucharczyk J, Stylopoulos N. Resting energy expenditure and energetic cost of feeding are augmented after Roux-en-Y gastric bypass in obese mice. Endocrinology 2012; 153(5): 2234-44. [PubMed: 22416083] 
19. Liou AP, Paziuk M, Luevano JM Jr., Machineni S, Turnbaugh PJ, Kaplan LM. Conserved shifts in the gut microbiota due to gastric bypass reduce host weight and adiposity. Sci Transl Med 2013; 5(178): 178ra41.

20. Bake T, Baron J, Duncan JS, Morgan DGA, Mercer JG. Arcuate nucleus homeostatic systems reflect blood leptin concentration but not feeding behaviour during scheduled feeding on a high-fat diet in mice. J Neuroendocrinol 2017; 29(8).

21. Romanova IV, Ramos EJ, Xu Y, Quinn R, Chen C, George ZM et al. Neurobiologic changes in the hypothalamus associated with weight loss after gastric bypass. J Am Coll Surg 2004; 199(6): 88795. [PubMed: 15555972]

22. Shin AC, Zheng H, Townsend RL, Sigalet DL, Berthoud HR. Meal-induced hormone responses in a rat model of Roux-en-Y gastric bypass surgery. Endocrinology 2010; 151(4): 1588-97. [PubMed: 20179262]

23. Chandarana K, Gelegen C, Karra E, Choudhury AI, Drew ME, Fauveau V et al. Diet and gastrointestinal bypass-induced weight loss: the roles of ghrelin and peptide YY. Diabetes 2011; 60(3): 810-8. [PubMed: 21292870]

24. Thaler JP, Yi CX, Schur EA, Guyenet SJ, Hwang BH, Dietrich MO et al. Obesity is associated with hypothalamic injury in rodents and humans. J Clin Invest 2012; 122(1): 153-62. [PubMed: 22201683]

25. Kirchner H, Hofmann SM, Fischer-Rosinsky A, Hembree J, Abplanalp W, Ottaway N et al. Caloric restriction chronically impairs metabolic programming in mice. Diabetes 2012; 61(11): 2734-42. [PubMed: 22787140]

26. Dalvi PS, Chalmers JA, Luo V, Han DY, Wellhauser L, Liu Y et al. High fat induces acute and chronic inflammation in the hypothalamus: effect of high-fat diet, palmitate and TNF-alpha on appetite-regulating NPY neurons. Int J Obes (Lond) 2017; 41(1): 149-158. [PubMed: 27773938]

27. Ahima RS, Prabakaran D, Mantzoros C, Qu D, Lowell B, Maratos-Flier E et al. Role of leptin in the neuroendocrine response to fasting. Nature 1996; 382(6588): 250-2. [PubMed: 8717038]

28. Korner J, Savontaus E, Chua SC Jr., Leibel RL, Wardlaw SL. Leptin regulation of Agrp and Npy mRNA in the rat hypothalamus. J Neuroendocrinol 2001; 13(11): 959-66. [PubMed: 11737554]

29. Marsh DJ, Miura GI, Yagaloff KA, Schwartz MW, Barsh GS, Palmiter RD. Effects of neuropeptide $\mathrm{Y}$ deficiency on hypothalamic agouti-related protein expression and responsiveness to melanocortin analogues. Brain Res 1999; 848(1-2): 66-77. [PubMed: 10612698]

30. Mizuno TM, Makimura H, Silverstein J, Roberts JL, Lopingco T, Mobbs CV. Fasting regulates hypothalamic neuropeptide Y, agouti-related peptide, and proopiomelanocortin in diabetic mice independent of changes in leptin or insulin. Endocrinology 1999; 140(10): 4551-7. [PubMed: 10499510]

31. Mizuno TM, Mobbs CV. Hypothalamic agouti-related protein messenger ribonucleic acid is inhibited by leptin and stimulated by fasting. Endocrinology 1999; 140(2): 814-7. [PubMed: 9927310]

32. Shimizu-Albergine M, Ippolito DL, Beavo JA. Downregulation of fasting-induced cAMP response element-mediated gene induction by leptin in neuropeptide $\mathrm{Y}$ neurons of the arcuate nucleus. $\mathrm{J}$ Neurosci 2001; 21(4): 1238-46. [PubMed: 11160394]

33. Boswell T, Nicholson MA, Bunger L. Neuropeptide Y gene expression in lines of mice subjected to long-term divergent selection on fat content. J Mol Endocrinol 1999; 23(1): 77-83. [PubMed: 10425449]

34. Palou M, Sanchez J, Rodriguez AM, Priego T, Pico C, Palou A. Induction of NPY/AgRP orexigenic peptide expression in rat hypothalamus is an early event in fasting: relationship with circulating leptin, insulin and glucose. Cell Physiol Biochem 2009; 23(1-3): 115-24. [PubMed: 19255506]

35. Xu B, Kalra PS, Farmerie WG, Kalra SP. Daily changes in hypothalamic gene expression of neuropeptide Y, galanin, proopiomelanocortin, and adipocyte leptin gene expression and secretion: effects of food restriction. Endocrinology 1999; 140(6): 2868-75. [PubMed: 10342879]

36. Champy MF, Selloum M, Piard L, Zeitler V, Caradec C, Chambon P et al. Mouse functional genomics requires standardization of mouse handling and housing conditions. Mamm Genome 2004; 15(10): 768-83. [PubMed: 15520880] 
37. Swoap SJ, Gutilla MJ, Liles LC, Smith RO, Weinshenker D. The full expression of fasting-induced torpor requires beta 3-adrenergic receptor signaling. J Neurosci 2006; 26(1): 241-5. [PubMed: 16399693] 
A
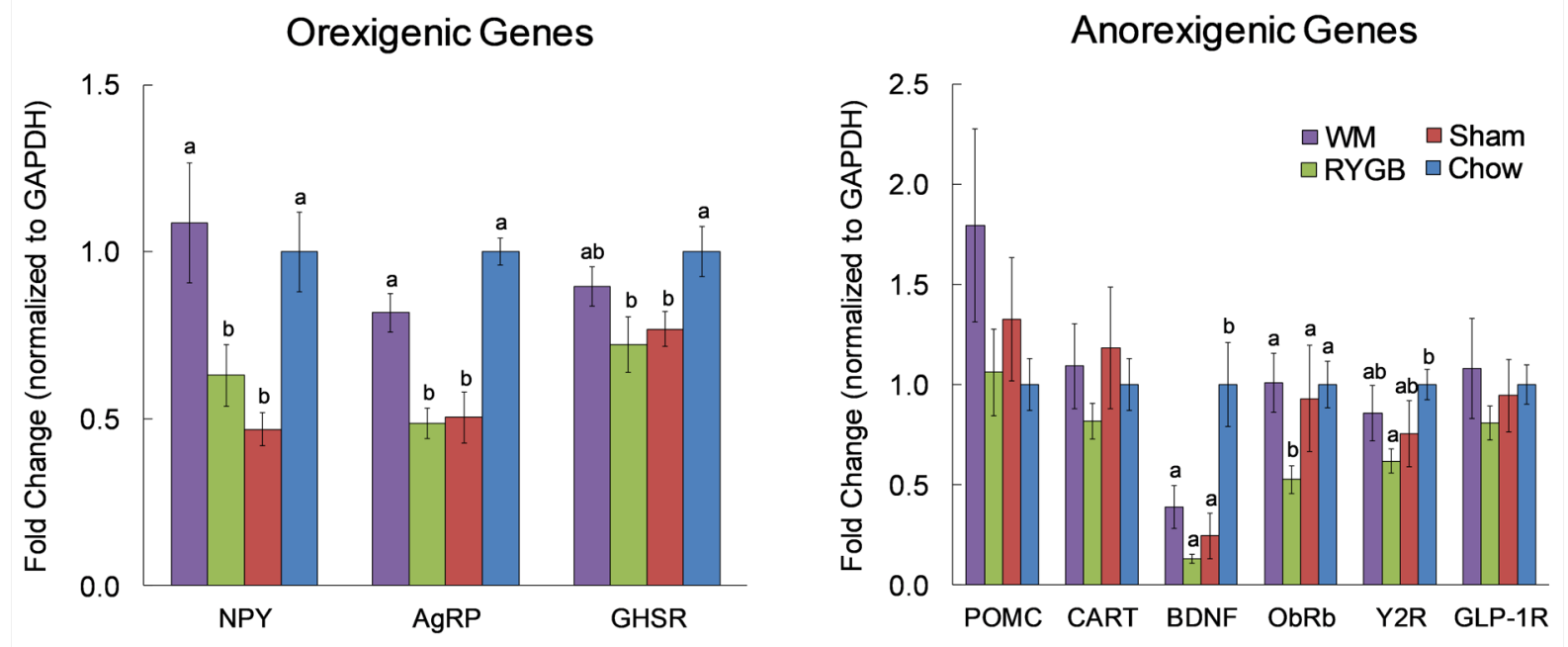

B

\section{WEEKS}

\section{Orexigenic Genes}

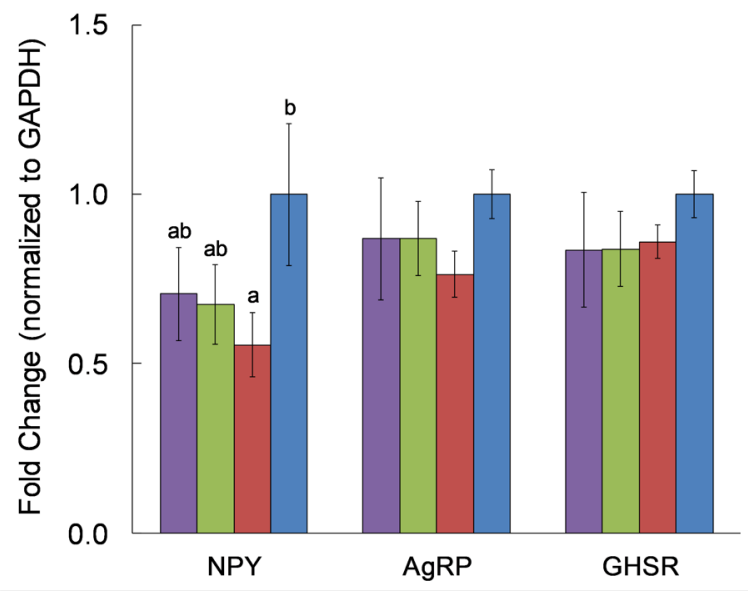

Anorexigenic Genes

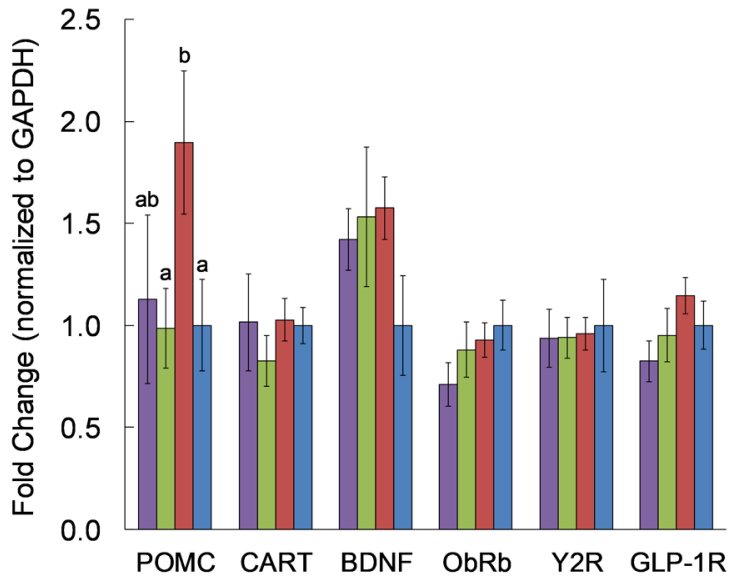

Fig. 1. Effects of RYGB surgery on mRNA abundance of orexigenic and anorexigenic genes in the MBH.

One cohort was sacrificed at 2 weeks (acute; Sham n=10; RYGB n=13; WM n=8; Chow $\mathrm{n}=6$ ) and the other at 12 weeks (chronic; Sham n=10; RYGB n=9; WM n=8; Chow n=4) post-surgically. Expression of oregixenic genes and anorexigenic genes during A) acute "weight-loss" phase and B) chronic "weight-maintenance" phase. mRNA levels are first normalized to GAPDH and are expressed as fold change from the chow-fed lean control group. Per each gene, bars without letters are not different from each other, while bars that 
do not share the same letters are statistically significant from each other. Data are presented as Mean \pm SEM. $\mathrm{p}<0.05$ based on one-way ANOVA followed by Bonferroni post-hoc test. 


\section{Inflammatory / Oxidative Stress Genes}
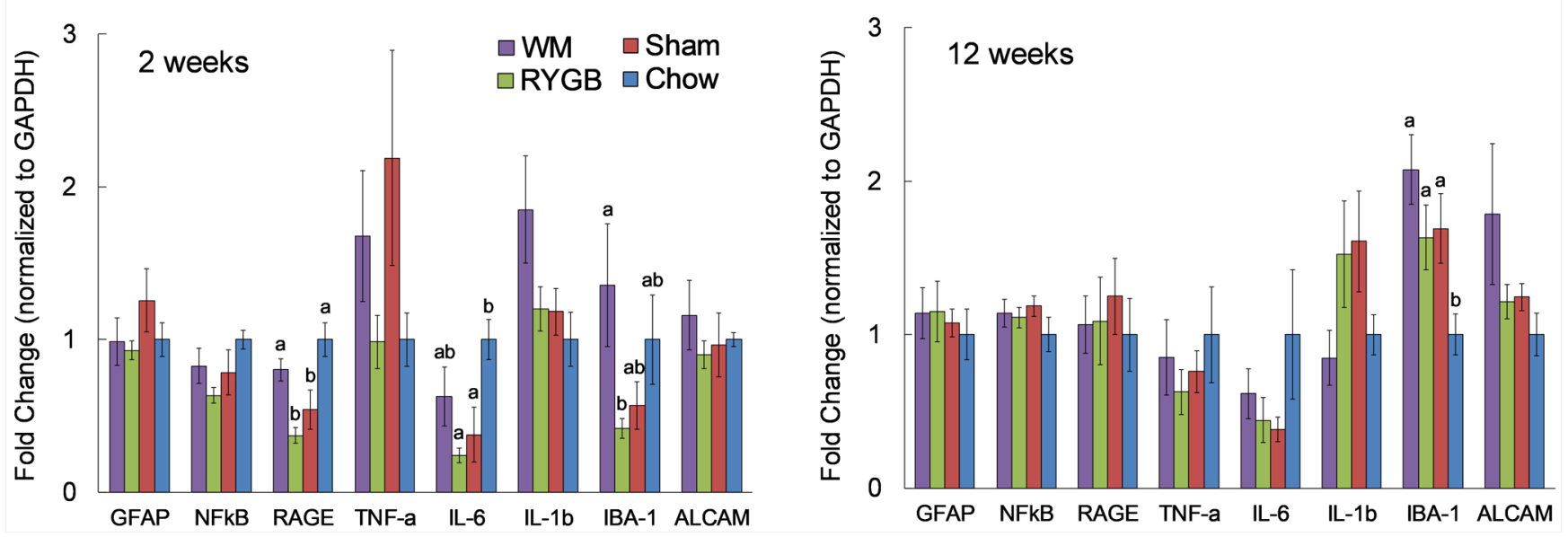

Fig. 2. Effects of RYGB surgery on mRNA abundance of genes in the MBH involved in inflammation and/or oxidative stress at 2 or 12 weeks post-surgery.

Per each gene, bars without letters are not different from each other, while bars that do not share the same letters are statistically significant from each other. Data are presented as Mean \pm SEM. $p<0.05$ based on one-way ANOVA followed by Bonferroni post-hoc test. 